\title{
Identification of PLK1 as a New Therapeutic Target in Mucinous Ovarian Carcinoma
}

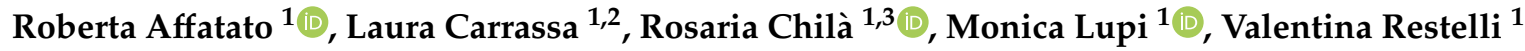 \\ and Giovanna Damia ${ }^{1, *}$ \\ 1 Laboratory of Molecular Pharmacology, Istituto di Ricerche Farmacologiche Mario Negri-IRCCS, \\ Via Mario Negri 2, 20156 Milan, Italy; roberta.affatato@marionegri.it (R.A.); laura.carrassa@unifi.it (L.C.); \\ rosaria.chila@ifom.eu (R.C.); monica.lupi@marionegri.it (M.L.); r.vale90@hotmail.it (V.R.) \\ 2 Core Research Laboratory-ISPRO, Viale Pieraccini 6, 50139 Firenze, Italy \\ 3 Istituto FIRC di Oncologia Molecolare (IFOM)—Via Adamello 16, 20139 Milan, Italy \\ * Correspondence: giovanna.damia@marionegri.it; Tel.: +39-0239-01-4234; Fax: +39-023-546-277
}

Received: 21 February 2020; Accepted: 11 March 2020; Published: 13 March 2020

check for updates

\begin{abstract}
Mucinous epithelial ovarian cancer ( $\mathrm{mEOC}$ ) is a rare subset of epithelial ovarian cancer. When diagnosed at a late stage, its prognosis is very poor, as it is quite chemo-resistant. To find new therapeutic options for $\mathrm{MEOC}$, we performed high-throughput screening using a siRNA library directed against human protein kinases in a mEOC cell line, and polo-like kinase1 (PLK1) was identified as the kinase whose downregulation interfered with cell proliferation. Both PLK1 siRNA and two specific PLK1 inhibitors (onvansertib and volasertib) were able to inhibit cell growth, induce apoptosis and block cells in the G2/M phase of the cell cycle. We evaluated, in vitro, the combinations of PLK1 inhibitors and different chemotherapeutic drugs currently used in the treatment of mEOC, and we observed a synergistic effect of PLK1 inhibitors and antimitotic drugs. When translated into an in vivo xenograft model, the combination of onvansertib and paclitaxel resulted in stronger tumor regressions and in a longer mice survival than the single treatments. These effects were associated with a higher induction of mitotic block and induction of apoptosis, similarly to what was observed in vitro. These data suggest that the combination onvansertib/paclitaxel could represent a new active therapeutic option in $\mathrm{mEOC}$.
\end{abstract}

Keywords: screening; PLK1; mucinous ovarian carcinoma

\section{Introduction}

Epithelial ovarian cancer (EOC) is the most lethal gynecological cancer, with about 239,000 new cases and 152,000 associated deaths worldwide annually [1,2]. Despite significant advances in surgical procedures and chemotherapy over the last two decades, the five year survival rate is lower than $50 \%$ for all stages and approximately $25 \%$ for advanced stage patients [3]. The high mortality is due to late diagnosis and primary or acquired resistance to therapy [4]. While histological and molecular data have clearly demonstrated that EOC is a heterogeneous disease, patients are still treated as a homogeneous group with cyto-reductive surgery followed by platinum/taxane doublet therapy [5,6]. Amongst the five histotypes (high-grade serous (ovarian cancer)—HGSOC, clear-cell, endometrioid, mucinous and low-grade serous), mucinous epithelial ovarian cancer (mEOC) represents approximately $3 \%$ of EOC and is classified as a Type I (low grade) tumor $[7,8]$. Most patients $(80 \%)$ with $\mathrm{mEOC}$ are diagnosed at an early stage and have a good prognosis; however, advanced mEOC (Stage III/IV) has a very dismal one, with a median overall survival (OS) of 12-14 months, well below the 37-42 months OS observed in patients with non-mucinous epithelial ovarian cancer [5,7]. This is probably due to the fact that $\mathrm{mEOC}$ is poorly responsive to platinum-based chemotherapy compared to the other EOC 
histological subtypes $[5,9]$. In fact, only $\approx 30 \%$ of patients respond to first line therapy, whereas $70 \%$ of high-grade serous ovarian cancer (HGSOC) patients do [10,11]. mEOC is also different from other subtypes at the molecular level. In fact, only $16-52 \%$ of mEOCs have a TP53 mutation compared to $99 \%$ of HGSOCs $[12,13]$. mEOC is not associated with $B R C A 1 / 2$ mutations, whereas approximately $25 \%$ of HGSOCs carry either germline or somatic mutations of these genes [12,14]. KRAS mutations are observed in $40-50 \%$ of patients, and ERBB2 (human epidermal growth factor receptor 2, HER2) gene amplification has been observed in $20-30 \%$ of invasive mEOCs $[15,16]$. Furthermore, mEOC has been associated with a homozygous loss of the cyclin-dependent kinase inhibitor 2A (CDKN2A) locus, and mutations in genes that activate the mitogen activated protein kinase (MAPK) pathways (such as $B R A F, A K T 1$ and PI3K) [13].

A better understanding of $\mathrm{mEOC}$ biology and more specific and active treatments are required to improve the prognoses of these patients. However, the rarity of the disease renders the preclinical and clinical research on this tumor type difficult [12]. Recent improvements in high-throughput screening and the availability of RNA interference (RNAi) libraries have demonstrated the power of this approach for target discovery and validation $[17,18]$. With the aim of identifying new therapeutic targets in $\mathrm{mEOC}$, we performed high-throughput screening with a piece of technology validated in our laboratory [19] and a siRNA library targeting 719 human kinases in a mEOC cell line. We were able to identify polo-like kinase 1 (PLK1), a mitotic serine/threonine kinase, as a potential therapeutic target in mEOC. We explored the effects of PLK1 inhibitors in mucinous ovarian cancer cell lines, either as single agents or in combination with the chemotherapeutic drugs used as standard therapy for treatment of $\mathrm{mEOC}$, and found that the combination of onvansertib (a PLK1 inhibitor) and paclitaxel was extremely active both in vitro and in vivo.

\section{Materials and Methods}

\subsection{Cell Culture and Drugs}

MCAS cells were grown in MEM (minimum essential medium) with 1\% glutamine and 20\% fetal bovine serum. EFO27 was grown in RPMI1640 with 1\% glutamine and $10 \%$ fetal bovine serum. JHOM1 was grown in DMEM/HamF12 with $1 \%$ glutamine, $10 \%$ fetal bovine serum and 0.1 mM NEAA. All cell lines were maintained in a humidified $37^{\circ} \mathrm{C}$ incubator with $5 \% \mathrm{CO}_{2}$. Cell lines were obtained from ATCC, and their authentication has been carried out by the authors within the last 6 months.

The PLK1 inhibitors volasertib (Selleckchem) and onvansertib (kindly provided by Trovagene, Inc, San Diego, LA USA) were dissolved in DMSO as $10 \mathrm{mM}$ stock solutions and stored at $-20^{\circ} \mathrm{C}$ for in vitro studies. For in vivo studies, onvansertib was dissolved in vehicle $(0.5 \%$ methylcellulose Tween-20), freshly, on days of dosing. Cisplatin (CDDP, Sigma-Aldrich, Milan, Italy) was dissolved in medium before use. Paclitaxel (Indena S.p.a., Milan, Italy) was dissolved in DMSO for in vitro studies and stored at $-20^{\circ}$; for in vivo studies it was dissolved in 50\% CremophorEL (Sigma-Aldrich, St. Louis, MO, USA) and 50\% ethanol, and further diluted with saline before use [20]. A list of all the drugs used and their mechanisms of action is provided in Table S1.

\subsection{High-Throughput siRNA Screening}

The Mission siRNA Human Kinase Panel (Sigma Aldrich, St Louis, MO, USA) comprising 719 different targets, represented in three different siRNAs, was used. The lyophilized siRNAs were dissolved to a final concentration of $2 \mu \mathrm{M}$ in nuclease free water and the siRNAs targeting the same gene were pooled at equal molarity in 96-well plates. For the high-throughput screening, we used an automated liquid handling system (JANUS ${ }^{\mathrm{TM}}$, PerkinElmer, Waltham, MA, USA), connected to a WinPREP for Janus software. MCAS cells were seeded at 25,000 cell $/ \mathrm{mL}$ ( $25 \mu \mathrm{L} /$ well) in 384 well plates; the next day, cells were transfected with the siRNA pool $(60 \mathrm{nM})$ of each target or mission siRNA universal negative control included in the library. Lipofectamine $2000(0,05 \mu \mathrm{L} /$ well $)$ was used as transfection reagent. Ninety-six hours after transfection, cell survival was analyzed by MTS 
assay (Promega, Madison, Wisconsin, USA). MTS reagent $(5 \mu \mathrm{L})$ was added to the cells, and after a constant incubation time for all the plates, absorbance was acquired using a plate reader (Infinite M200, TECAN, Mannedorf, Switzerland). Each sample was transfected in triplicate, and the entire screening was repeated twice. The validation procedure was performed as follows: each of the three siRNAs of the pool was deconvoluted and transfected singly. A second validation was performed with specific esiRNAs (endoribonuclease-prepared siRNAs, Sigma Aldrich, St Louis, MO, USA) transfected with Lipofectamine 2000 (Invitrogen, Carlsbad, CA, USA) at a concentration of $50 \mathrm{nM}$, as already reported [20]. esiRNAs are a mixture of siRNA oligos targeting the same mRNA sequence, leading to highly specific and effective gene silencing. Negative control esiRNA was EGFP esiRNA.

\subsection{Western Blot Analysis}

Cells were lysed in ice-cold whole cell extract buffer containing $50 \mathrm{mM}$ TrisHCl at $\mathrm{pH} 7.4,250 \mathrm{mM}$ $\mathrm{NaCl}, 0.1 \%$ Nonidet NP40, $5 \mathrm{mM}$ EDTA and NaF $50 \mathrm{mM}$ with a protease inhibitor cocktail (Sigma). Lysates were cleared by centrifuging at 12,000 rpm for $5 \mathrm{~min}$. Cell lysates containing equal amounts of protein $(30-70 \mu \mathrm{g})$ were resolved on 10-12\% SDS-PAGE (polyacrylamide gel electrophoresis) gels. The proteins were then transferred to nitrocellulose membranes (PROTRAN, Schleicher and Shull). Immunoblotting was carried out with the following antibodies and visualized using Odissey FC Imaging System (Li-COR): anti-PLK1 (F-8) \#sc17783, anti-actin (C-11) \#sc1615 and anti-beta tubulin \#sc9104 were provided by Santa Cruz Biotechnology. Anti-phospho-Histone H3 (Ser10) (6G3) \#9706 was purchased from Cell Signaling Technology and anti-H2AX pSer139 \#05-636 was purchased by Millipore. The horseradish peroxidase (HRP) conjugated secondary antibody anti-goat (sc-2354) was purchased from Santa Cruz Biotechnology (Heidelberg, Germany). The anti-rabbit (\#1706515) and anti-mouse (\#1706516) antibodies were purchased from BIO-RAD LABORATORIES S.r.l.

\subsection{Cell Viability Assay}

To validate the effect of PLK1 downregulation in mucinous ovarian cancer cell lines, MCAS and EFO27 were seeded in 96 well plates, and after $24 \mathrm{~h}$ were transfected with specific esiRNA of PLK1 (50nM) by Lipofectamine 2000. Cell viability was measured 48 and $72 \mathrm{~h}$ after transfection by MTS assay. To evaluate the cytotoxic effect of PLK1 inhibitors, cell lines were seeded in 96 well plates at densities of 10,000 cell/mL for MCAS and JHOM1, and 15,000 cell/mL for EFO27 in 10\% fetal bovine serum medium. After $48 \mathrm{~h}$, cells were treated with serial dilution of onvansertib or volasertib, and $72 \mathrm{~h}$ later cell viability was assessed by MTS assay. Drug-dose response curves were generated and IC50 calculated by using Prism7.05 (GraphPad Software, San Diego, CA, USA). To assess the effect of the combination of PLK1 inhibitors (onvansertib and volasertib) with cisplatin and/or paclitaxel and/or eribulin and/or PI3K inhibitor (PIK75), cells were seeded, and $48 \mathrm{~h}$ after, treated simultaneously with a growing concentration of the drugs, and cell viability was measured $72 \mathrm{~h}$ after by MTS assay. Results were examined by isobologram analysis with Calcusyn Software (Biosoft, Cambridge, UK), and combination index $(\mathrm{CI})$ values at the $\mathrm{IC}_{50}$ were calculated to assess the efficacy of the combination, as already reported [21]. All the experiments were performed in triplicate and repeated at least twice.

\subsection{Flow Cytometry}

For flow cytometric analysis of DNA content, cells were fixed in ice-cold $70 \%$ ethanol; washed in PBS; resuspended in $1 \mathrm{~mL}$ of $25 \mu \mathrm{g} / \mathrm{mL}$ of propidium iodide and $12.5 \mu \mathrm{L}$ of RNase $(1 \mathrm{mg} / \mathrm{mL})$; and stained for $2 \mathrm{~h}$ at room temperature in the dark. Cell cycle analysis was done on at least 10,000 cells for each sample using the FACS Calibur (Becton Dickinson, Franklin Lakes, NJ, USA) [21].

For two-parameter flow cytometry analysis of DNA content and p-S10 histone H3, about $1 \times 10^{6}$ cells fixed in ethanol 70\% were washed with PBS and permeabilized in Triton X-100 $0.25 \%$ in PBS for $10 \mathrm{~min}$ on ice. Then, cells were washed and incubated with $100 \mu \mathrm{L}$ of anti p-S10 histone H3 (Cell Signaling Technology \#9706) diluted 1:100 in PBS containing 0.5\% BSA for $2 \mathrm{~h}$ at room temperature. 
After washing with PBS, cells were incubated with Alexa-fluor488 (goat anti-mouse, Molecular Probe \#A-11017) diluted 1:500 in PBS + 0.5\% BSA for $1 \mathrm{~h}$ at room temperature. After the incubation with antibody, cells were centrifuged, resuspended in $2.5 \mu \mathrm{g} / \mathrm{mL}$ PI in PBS plus $25 \mu \mathrm{L}$ of $1 \mathrm{mg} / \mathrm{mL}$ RNase in water, incubated overnight and analyzed.

\subsection{Caspase-3 Activity Assay}

Caspase-3 activity was measured by an enzymatic assay using a fluorogenic substrate for caspase-3, Ac-DEVD-AMC (acetyl Asp-Glu-Val-Asp 7-amido-4-methylcoumarin). MCAS protein extracts were collected after 24 and $48 \mathrm{~h}$ of treatment with onvansertib $15 \mathrm{nM}$, paclitaxel $2 \mathrm{nM}$ or both. They were mixed with the apoptosis buffer (Hepes pH $7.520 \mathrm{mM}$, glycerol 10\%, DTT $10 \mathrm{mM}$ ) in a white 96-well plate and incubated at $37^{\circ} \mathrm{C}$ for $5 \mathrm{~min}$. The substrate was then added at a final concentration of $12.5 \mu \mathrm{M}$. Fluorescent AMC production was measured at excitation $370 \mathrm{~nm}$ and emission $460 \mathrm{~nm}$ wavelengths, using a plate reader (Infinite M200, TECAN). The caspase-3 activity of each sample was examined in duplicate and expressed as the linear change in fluorescence units per hour and normalized for the protein concentration.

\subsection{Xenograft Model}

Five-week-old female NCr-nu/nu mice were obtained from Envigo Laboratories (Italy), and maintained under specific pathogen-free conditions, housed in isolated vented cages and handled using aseptic procedures. Procedures involving animals were conducted in conformity with the following laws, regulations and policies governing the care and use of laboratory animals: Italian Governing Law ( Decreto Legge 26/2014; authorization number 19/2008-A issued 6 March 2008 by the Ministry of Health); Mario Negri Institutional Regulations and Policies providing internal authorization for persons conducting animal experiments (Quality Management System Certificate: UNI EN ISO 9001:2008, regulation number 6121); the NIH Guide for the Care and Use of Laboratory Animals (2011 edition); and the EU directive and guidelines (EEC Council Directive 2010/63/UE). An institutional review board and the Italian Ministry of Health approved all the in vivo experiments performed with PDXs. Exponentially growing MCAS cells (approximately $5 \times 10^{6}$ cells per mouse) were injected subcutaneously. Animals were randomized nine/group when tumors reached approximately $120 \mathrm{mg}$ in different experimental groups (control, onvansertib, paclitaxel and combination). Onvansertib was given orally at the dose of $50 \mathrm{mg} / \mathrm{kg}$ for 4 consecutive days for 3 cycles with a 3 days rest, while paclitaxel was administered intravenously at the dose of $20 \mathrm{mg} / \mathrm{kg}$ once a week for 3 weeks. In the combined treatment, paclitaxel was administered $2 \mathrm{~h}$ after the last onvansertib treatment dose. Tumor growth was measured twice weekly with a Vernier caliper, and tumor weights $\left(\mathrm{mg}=\mathrm{mm}^{3}\right)$ were calculated as follows: (length $(\mathrm{mm}) \times$ width $\left.^{2}\left(\mathrm{~mm}^{2}\right)\right) / 2$ where width $<$ length); body weights were registered and considered an indirect parameter of drug toxicity. The efficacy of treatment was evaluated using the best tumor growth inhibition $(\% \mathrm{~T} / \mathrm{C}=$ (mean tumor weight of treated tumors/mean tumor weight of control tumors) $\times 100$ ). A T/C value $<42 \%$ is considered the minimum for antitumoral activity, according to published criteria [22]. For pharmacodynamic studies, tumor bearing mice were treated with single or combined drugs following the schedule, doses and routes used in the experiment; however, mice were treated for just one cycle and then sacrificed $24 \mathrm{~h}$ after the last treatment with onvansertib in one group, and $24 \mathrm{~h}$ after paclitaxel treatment in both paclitaxel single treatment group and in the combination groups. The tumors were removed and snap-frozen. The frozen samples were homogenized in protein lysis buffer, loaded on SDS-PAGE, and immunoblotted.

\subsection{Statistical Analysis}

Statistical significance was determined with GraphPad Prism7.05 (GraphPad Software, San Diego, CA, USA). Figures legends specify which test was used. 


\subsection{Ethics Approval and Consent to Participate}

Procedures involving animals were conducted in conformity with the following laws, regulations and policies governing the care and use of laboratory animals: Italian Governing Law (Decreto legge 26/2014; authorization no.19/2008-A issued 6 March 2008 by the Ministry of Health); Mario Negri Institutional Regulations and Policies providing internal authorization for persons conducting animal experiments (Quality Management System Certificate: UNI EN ISO 9001:2008, regulation number 6121); an institutional review board and the Italian Ministry of Health approved all the in vivo experiments performed with PDXs (project authorization \#9F5F5.69.EXT28).

\section{Results}

\subsection{High-Throughput siRNA Screening Identified PLK1 as a Potential Target}

We performed a high-throughput screening using a siRNA library targeting 719 human kinases in the MCAS cell line, and the effect of downregulation of each individual siRNA on cell growth was calculated by dividing the mean absorbance in the three replica wells transfected with siRNA (treated sample-T) by the mean absorbance of the replica wells transfected with a control/negative siRNA (control sample-C) (T/C ratio). Two independent experiments were run and a preselected, arbitrary $\mathrm{T} / \mathrm{C}$ cut-off value of 0.6 , corresponding to $40 \%$ of inhibition of cell growth was chosen. Following those criteria, 17 hits with a mean of $\mathrm{T} / \mathrm{C} \leq 0.6$ in the two experiments were found (Figure 1A, Table S2). We then validated 13 out of the 17 positive hits with specific esiRNAs, and only polo-like kinase1 (PLK1) esiRNA, a mitotic serine/threonine kinase, was able to significantly interfere with cell survival (Figure 1B). Concerning the two other members of the polo-like kinase family, PLK2 and PLK3, they had no effect on cell survival $(\mathrm{T} / \mathrm{C}>0.6)$.

A

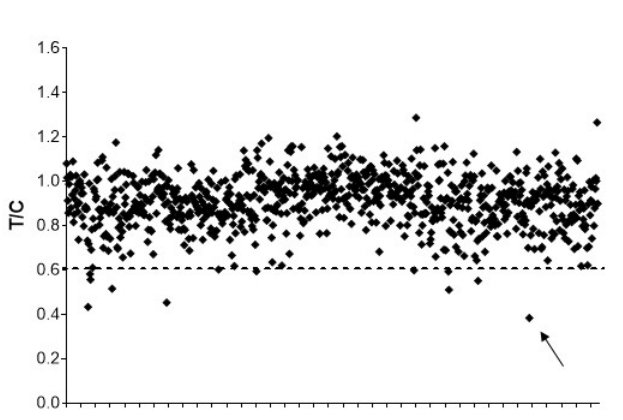

B

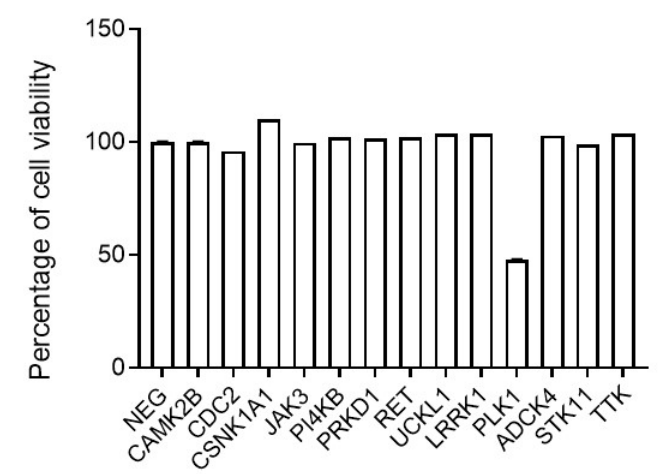

Figure 1. High-throughput siRNA screening in the mucinous epithelial ovarian cancer (mEOC) cell line MCAS. (A) Summary of the effects of downregulation on each member of the 719-in-size target pool of the siRNA library. Data are plotted as ratios of transfected siRNA/negative control siRNA mean absorbance values $(\mathrm{T} / \mathrm{C})$ and represent the means of the independent siRNA screenings done. The dashed line selected 17 siRNA with a T/C $\leq 0.6$. The arrow underlines the T/C value of PLK1. (B) Validation of siRNA screening. MCAS cell survival after $96 \mathrm{~h}$ of transfection using specific esiRNA against positive hits identified in the screening.

\subsection{The Effect of PLK1 Inhibition in Mucinous Ovarian Cancer Cell Lines}

To further investigate the effect of downregulation of PLK1, two mucinous ovarian cancer cell lines (MCAS and EFO27) were transiently transfected with negative esiRNA or PLK1 esiRNA, and cell viability, cell cycle phase distribution and apoptosis were evaluated. PLK1 specific esiRNA strongly inhibited the expression of PLK1 in both MCAS and EFO27 (Figure 2A) and caused a strong inhibition in cell growth compared to the cells transfected with negative esiRNA (Figure 2B). Cell cycle analysis showed an accumulation of cells in the G2/M phase and a slight increase in the sub-G1 population 48 
and $72 \mathrm{~h}$ after esiRNA PLK1 transfection, while no modification of cell cycle distribution was observed after transfection with negative esiRNA (Figure 2C). A small number of polyploid cells, indicative of endoreduplication, were detected at $72 \mathrm{~h}$ in EFO27, but not among MCAS cells. Sub-G1 population is suggestive of cell death, and indeed, PLK1 depletion was able to induce apoptosis as measured by activation of caspase-3 (Figure 2D).

A

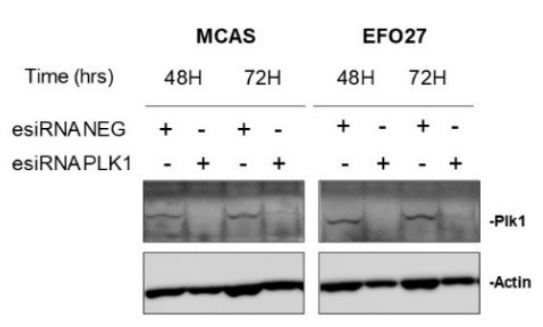

C

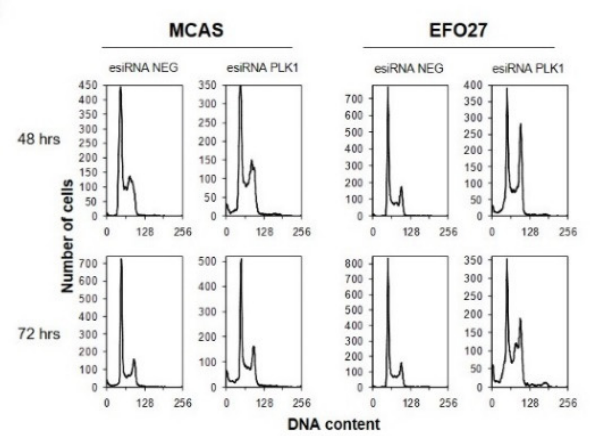

B
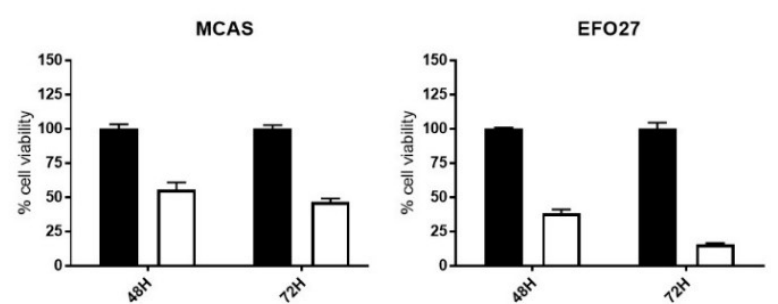

D

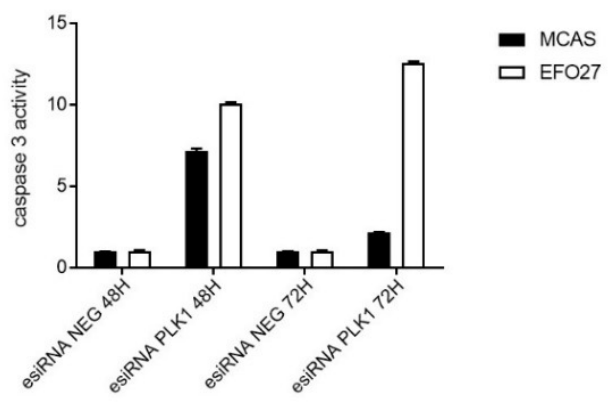

Figure 2. PLK1 downregulation in MCAS and EFO27 cell lines. (A) Western blot analysis showing PLK1 downregulation after transfection with esiRNA in MCAS and EFO27 cell lines. (B) Percentages of cell viability at 48 and $72 \mathrm{~h}$ after esiRNA transfection. Black bar, negative esiRNA; white bar, PLK1 esiRNA. (C) Flow cytometric analysis of DNA content. (D) Caspase-3 activity in MCAS (black bar) and EFO27 (white bar) cell lines.

We then moved from esiRNA mediated PLK1 downregulation to its chemical inhibition. To do this, we evaluated the cytotoxic activity of commercially available PLK1 inhibitors (volasertib and onvansertib) as single agents in three mEOC cell lines (MCAS, EFO27, JHOM1) and found both drugs very active at nanomolar concentrations; similar cytotoxic effects were observed in non-mucinous ovarian cancer cells (Table 1).

Table 1. Onvansertib and volasertib IC50s (nM) in the different ovarian cancer cell lines.

\begin{tabular}{ccc}
\hline Mucinous Cell Lines & Onvansertib (nM) & Volasertib (nM) \\
\hline MCAS & $48 \pm 6$ & $16 \pm 2$ \\
\hline EFO27 & $287.9 \pm 22$ & $71 \pm 7$ \\
\hline JHOM1 & $87.7 \pm 11$ & $13.6 \pm 4$ \\
\hline Serous cell lines & \\
\hline A2780 & $47 \pm 4.8$ & $13.5 \pm 1.9$ \\
\hline OVCAR5 & $65.9 \pm 16$ & $28.8 \pm 5.6$ \\
\hline SKOV3 & $132.1 \pm 25$ & $44.2 \pm 11$ \\
\hline
\end{tabular}

Onvansertib treatment in MCAS cells at the dose of $\mathrm{IC}_{50}$ induced effects on the cell cycle similar to those observed with esiRNA anti-PLK1. A block in G2/M phase and cell death, suggested by the presence of sub-G1 events, were already present at $24 \mathrm{~h}$ and persisted until $48 \mathrm{~h}$. In EFO27 cells, 
onvansertib treatment led the cells to duplicate their DNA, inhibiting the cytokinesis and inducing the formation of polyploid cells (Figure S1). JHOM1 cells showed a drug induced cell cycle perturbation similar to that induced in MCAS cells.

\subsection{Investigation of Synergistic Combination with PLK1 Inhibitors}

We then looked for potential synergistic combinations of PLK1 inhibitors with drugs used in first line treatment of ovarian carcinoma (cisplatin and paclitaxel) and with other investigational drugs (eribulin and PI3K inhibitors). No synergism could be found in any of the cell lines, when both PLK1 inhibitors were combined with cisplatin (Figure S2). On the contrary, strong synergism could be observed in two out of the three mEOC cell lines (MCAS and JHOM1 cells) when onvansertib (Figure 3A,B respectively) and volasertib (Figure S3A,B) were combined with both paclitaxel and eribulin, with CI lower than 1 (Figures 3D,E and S3D,E). In EFO27 cell line, the least sensitive of PLK1 inhibitors, no synergistic activity was observed with onvansertib and volasertib (Figures 3C-E and S3C-E).

A

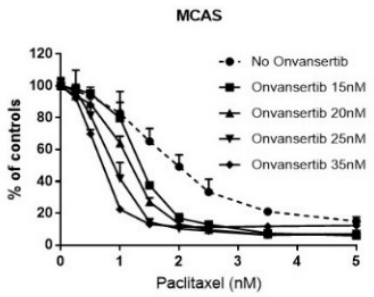

B
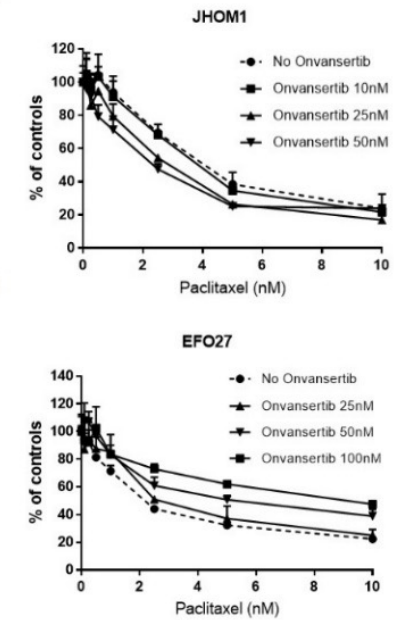
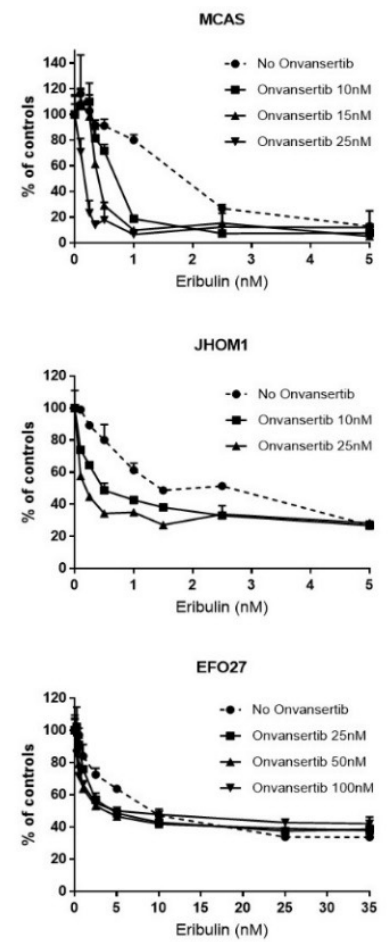

D

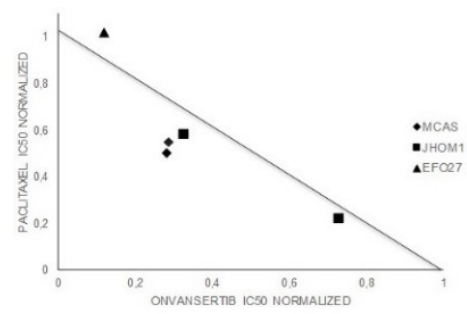

E

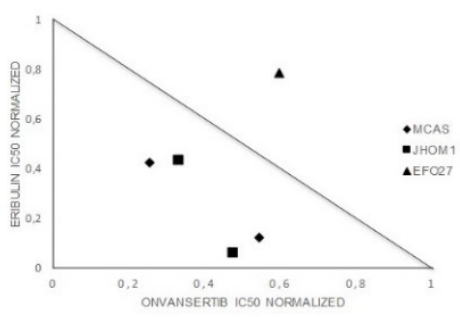

Figure 3. Drug combination of onvansertib and paclitaxel or eribulin in mEOC cell lines. (A-C) MCAS, JHOM1 and EFO27 treated with non-toxic concentrations of onvansertib and increasing concentrations of paclitaxel and eribulin. (D,E) normalized IC50 isobolograms showing the synergistic effects of the combination of onvansertib with paclitaxel and eribulin in MCAS and JHOM1 cell lines and the antagonistic effect in EFO27. Each symbol represents an independent experiment.

MAPK pathway deregulation has been reported in mucinous ovarian cancer, and our cell lines are representative of mEOC in terms of pathway deregulation (Table S3). The combination of volasertib/onvansertib and PIK75 (a PI3K inhibitor) was found to be synergic in all the considered cell lines (Figure S4).

\subsection{Molecular Studies on the Basis of the Synergistic Onvansertib/Paclitaxel Combination}

Considering that paclitaxel is a drug approved for the treatment of mucinous ovarian carcinoma, we focused on onvansertib/paclitaxel combination. To investigate the mechanism of the synergistic cytotoxic effect, MCAS cells were treated for 8, 24 and $48 \mathrm{~h}$ with low concentrations of onvansertib $(15 \mathrm{nM})$ and paclitaxel $(2 \mathrm{nM})$ alone or in combination. As shown in Figure $4 \mathrm{~A}$, the combination caused 
a marked decrease in cell viability at 24 and $48 \mathrm{~h}$. Onvansertib alone at this concentration induced only a slight accumulation of cells in the G2/M phase after a short treatment time $(8 \mathrm{~h})$, while the same effect was more persistent in paclitaxel treated samples. The combined treatment caused a much higher block of cells in the G2/M phase, starting from $8 \mathrm{~h}$ and still present at $48 \mathrm{~h}$ (Figure 4B). Phospho-histone $\mathrm{H} 3$ staining confirmed a higher percentage of cells blocked in $\mathrm{M}$ phase of the cell cycle in the combined group compared to single onvansertib and paclitaxel treatment.

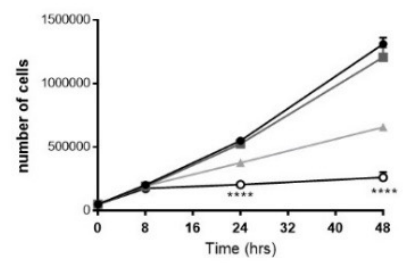

C

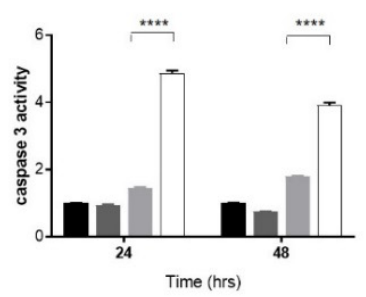

B
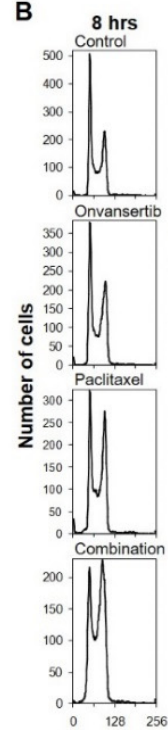
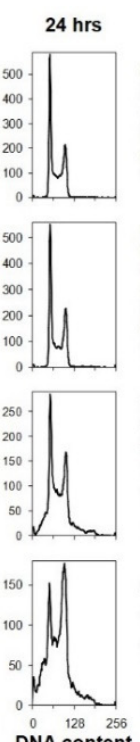
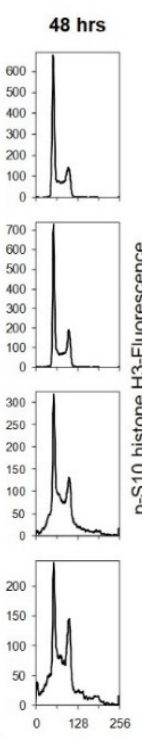
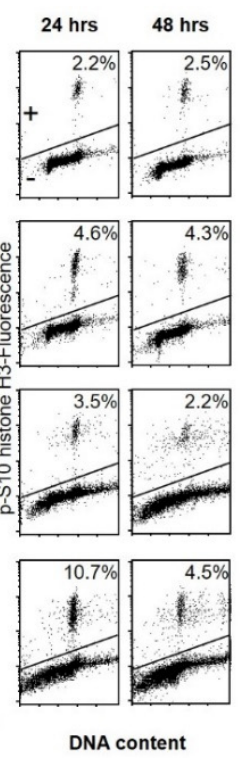

D
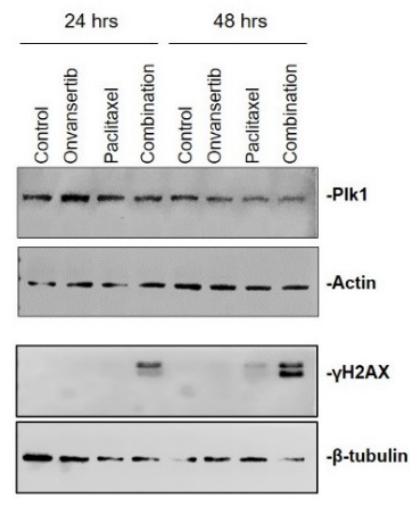

Figure 4. Effect of the combined treatment of onvansertib/paclitaxel on the MCAS cell line. (A) Cell

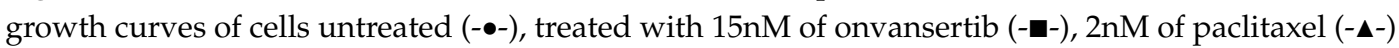
or the combination (-○-). Cell growth was followed from the day of treatment and up to $48 \mathrm{~h}$ after treatment. The data are expressed as number of cells $\pm \mathrm{SD}$ of three replicates. A two-way ANOVA test performed with GraphPad Prism was used for statistical analysis. Statistically significance differences are observed from 24 to $48 \mathrm{~h}$ in MCAS cell combined treatment groups vs. control and vs. single agents: ${ }^{* * *} p<0.0001$. (B) Analysis of DNA content after 8,24 and $48 \mathrm{~h}$ of treatment with the two drugs either single (paclitaxel $2 \mathrm{nM}$, onvansertib $15 \mathrm{nM}$ ) or combined, and biparametric analysis of pS10-histone H3 and DNA after 24 and $48 \mathrm{~h}$. Cells detected above the horizontal line were considered as pS10-histone H3-positive, and this percentage was reported in each plot. (C) Activation of caspase-3 by enzymatic assay 24 and $48 \mathrm{~h}$ after treatment. Data are percentages of untreated cells and represent the means \pm SDs. For statistical analysis, a two-way ANOVA test with Bonferroni multiple comparison was used, paclitaxel vs. combination $24 \mathrm{~h}^{* * * *} p<0.0001$, paclitaxel vs. combination $48 \mathrm{~h}^{* * * *} p<0.0001$. (D) Western blot analysis showing PLK1, $\gamma \mathrm{H} 2 \mathrm{AX}$, actin and beta-tubulin protein levels in MCAS cells protein extracts.

The combination treatment caused an increase of sub-G1 population at 24 and $48 \mathrm{~h}$, suggestive of cell death (Figure 4B), which was confirmed by the five-fold increase in caspase-3 activity observed in extracts of cells treated with the two drugs at 24 and $48 \mathrm{~h}$ compared to cells untreated or treated with the single agents (Figure 4C). The induction of apoptosis was also investigated at earlier time points, but almost no apoptosis could be seen (Figure S5A). Higher $\gamma \mathrm{H} 2 \mathrm{AX}$ levels were observed in cells treated with the combination than in control and single-treatment cells, only at 24 and $48 \mathrm{~h}$ (Figure 4D) and not at the earlier time points investigated (Figure S5B), suggesting that its increase is related to apoptosis induction. Similar results were obtained with the combination of onvansertib and eribulin at $48 \mathrm{~h}$ (Figure S6). We looked for possible pharmacodynamic markers of activity. As shown in Figure 4D, no difference was observed in PLK1 protein levels after treatment, either after single or combined treatments. 


\subsection{In Vivo Effects of the Combination}

On the basis of the strong in vitro synergistic effect, the combination onvansertib and paclitaxel was tested in vivo in nude mice transplanted with MCAS cells. To try to magnify the effect of the combination, we used a relatively low dose of onvansertib $(50 \mathrm{mg} / \mathrm{kg})$; indeed, at this dose, as a single agent the drug was inactive ( $\mathrm{T} / \mathrm{C}=78.5 \%$ ) and no tumor growth inhibition was observed (Figure $5 \mathrm{~A}$ ); paclitaxel at the dose of $20 \mathrm{mg} / \mathrm{kg}$ was able to induce tumor growth inhibition and some regressions. However, the combination of the two drugs, which was well tolerated (Figure S7), was much more active than paclitaxel as a single agent, as suggested by a stronger and more sustained tumor regression and tumor growth inhibition (Figure 5A), which translated in a longer survival time (Figure 5B). Pharmacodynamic analysis on tumors treated with drugs as single agents or a combination at the same doses used in the activity, showed a slight increase of caspase- 3 activation in tumor protein extracts from mice treated with the combination (Figure 5C) and a slight increase of the mitotic marker phospho-histone $\mathrm{H} 3$ and $\gamma \mathrm{H} 2 \mathrm{AX}$ after both paclitaxel and combined treatments (Figure 5D).

A

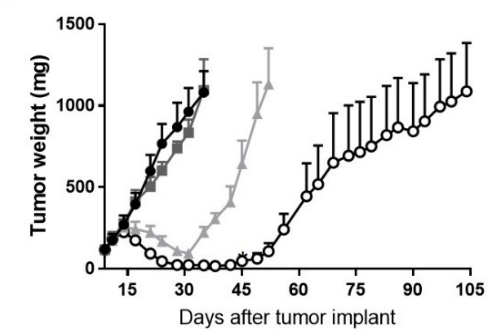

C

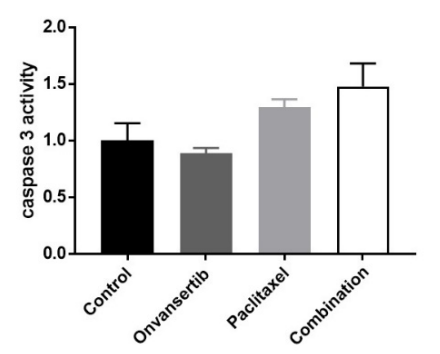

B

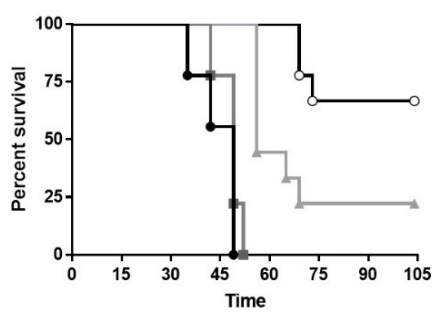

D

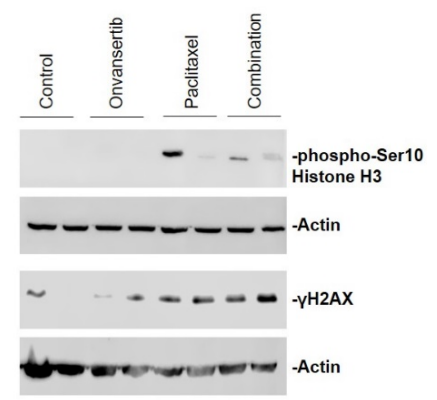

Figure 5. Antitumor effect and target modulation of the combined treatment in MCAS xenografts.

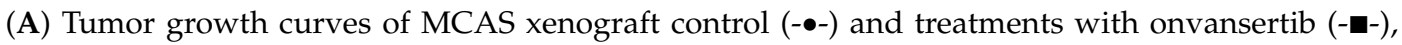
paclitaxel (-ム-) or both (-○-). Data are represented as means \pm SEs. For statistical analysis, two-way ANOVA test with Bonferroni multiple comparison was used. On day 35 the significant differences were as follows: control vs. paclitaxel and combination, ${ }^{* * *}, p<0.0001$; onvansertib vs. paclitaxel and combination, ${ }^{* * *}, p<0.0001$. One-way ANOVA test for repeated measures with Bonferroni multiple comparison was used for statistical analysis at day 45 and the significant differences were as follows: control vs. combination, ${ }^{* *}, p<0.01$; onvansertib vs. combination, ${ }^{* * * *}, p<0.0001$; paclitaxel vs. combination, ${ }^{*}, p<0.05$. (B) Kaplan-Meier survival curves of mice. Log-rank test (Mantel-Cox) was used to calculate $\mathrm{p}$-values comparing the survival curves. Control vs. paclitaxel and combination ${ }^{* * * *}, p<0.0001$; onvansertib vs. paclitaxel and combination ${ }^{* * * *}, p<0.0001$; paclitaxel vs. combination ${ }^{*}, p<0.01$. (C) Caspase 3 activity in tumor tissue extracts from mice treated as described in the methods. Data are percentages of untreated cells and represent the means \pm SDs of two independent experiments each. One-way ANOVA test with Tukey's multiple comparison was used, and the differences were as follows: control vs. combination ${ }^{*}, p<0.05$; onvansertib vs. paclitaxel ${ }^{*}, p<0.05$; onvansertib vs. combination $^{* *}, p<0.01$. (D) Western blot analysis showing pS10 Histone H3 and $\gamma \mathrm{H} 2 \mathrm{AX}$ protein levels in xenograft tumors protein extracts. Two replicates for each condition were used. 


\section{Discussion}

Advanced mucinous ovarian cancer is a chemo-resistant disease with a poor clinical outcome $[6,7,23]$. Therefore, the development of more specific and active therapeutic strategies for late stage $\mathrm{mEOC}$ are required to improve the survival of these patients. In order to find new therapeutic options for this rare cancer, we performed a high-throughput screening using a human siRNA kinase library in a mEOC cell line to identify genes interfering with cell growth/viability. After two independent screenings and validation experiments using specific esiRNAs, PLK1, belonging to the family of mitotic serine/threonine kinases, was identified as the most significant kinase whose downregulation caused a strong growth inhibiting effect in MCAS cell line.

PLK1 is the most investigated member of human PLK family and is essential for mitosis, as it regulates several stages of this phase of cell cycle (mitotic entry, centrosome maturation, mitotic spindle formation, transition from metaphase to anaphase and cytokinesis) [24,25]. Several studies have shown that PLK1 is overexpressed in a broad spectrum of malignant tumors, including ovarian cancer, and its expression correlates with histological grade and poor prognosis [26,27]. For all these reasons, in recent years, PLK1 has become an interesting therapeutic target in oncology [28-30], and several PLK1 inhibitors have indeed been developed and are under clinical investigation [31], including volasertib in phase III study in acute myeloid leukemia [32], and onvansertib, undergoing investigation in phase II in combination with other drugs (https://trovageneoncology.com/pipeline/). Of note, a recent study reported hints of antitumor activity in patients with platinum-resistant or -refractory ovarian cancer treated with volasertib, suggesting PLK1 could be an interesting target to be further investigated in ovarian cancer [33].

Based on the screening results, the specific inhibition of PLK1 with esiRNA in mEOC cell lines leads to cell growth inhibition, G2/M cell cycle blocking, induction of apoptosis and endoreduplication. While these data are consistent with previous findings of the inhibition of PLK1 using RNAi [34-36], this is the first observation that PLK1 inhibition is effective in mEOC. In addition, PLK1 inhibitors volasertib and onvansertib were cytotoxic at low nanomolar concentrations in all the $\mathrm{mEOC}$ cell lines tested, similarly to what has been reported in other tumor cells [37-39]. When we investigated the effect of volasertib and onvansertib treatment in combination with the chemotherapeutic drugs used as standard therapy for treatment of $\mathrm{mEOC}$, we did not find synergy with cisplatin in any of the mEOC cell lines tested; on the contrary, a synergistic effect was observed when onvansertib/volasertib were combined with microtubule-interacting drugs, paclitaxel and eribulin, in two out of three cell lines tested. The strong effect of the drug combination is demonstrated by a $\mathrm{CI}<1$, which corroborates a synergistic drug interaction. The lack of synergy in EFO27 cell line could be due to its lower sensitivity to both volasertib and onvansertib, as indicated by the 3-6 fold higher drug IC50s compared to the other mEOC cell lines. Some evidence suggests that high concentrations of PLK1 inhibitors might promote mitotic slippage favoring cell survival [40]. In time lapse experiments, EFO27 cells treated with onvansertib at different concentrations entered mitosis, but failed to undergo chromosome segregation and cytokinesis with the formation of polyploid and multinucleated cells (endoreduplicated cells) that might be more resistant to apoptosis induced by chemotherapy (Figure S1A, time lapse experiments-data not shown). In support of these data, when we tested the induction of apoptosis after paclitaxel/onvansertib treatment in EFO27 cell line, a much lower increase of apoptosis was observed when compared to MCAS cell lines (Figure S8). The lack of synergism with cisplatin is not surprising. The two drugs act on different targets in different phases of the cell cycle, and the G2 block induced by cisplatin would possibly downregulate the number of cells in $\mathrm{M}$ phase where onvansertib should maximize its activity.

We tried to elucidate the underlying molecular mechanisms of the synergism of PLK1 inhibitors with paclitaxel and found that the combined treatment causes strong growth inhibition and mitotic arrest, which triggers activation of caspase- 3 and apoptosis; these results correlate with previous findings $[36,41]$. Recently, Noack et al. identified a synthetic lethality between volasertib and paclitaxel in ovarian cancer cell lines with CCNE1-amplification, demonstrating that the combination induces 
a prolonged mitotic arrest and cell death, shifting the balance between pro- and anti-apoptotic proteins toward cell death [42].

Our in vitro data translated into a pronounced antitumor activity of the combination in the MCAS mEOC xenograft with a lack of evident toxicity. The reported in vitro molecular mechanisms were also observed in vivo, where in tumors treated with the combination showed a higher level of phospho-histone $\mathrm{H} 3$, indicative of a sustained mitotic block and a higher induction of $\gamma \mathrm{H} 2 \mathrm{AX}$, indicative of increased DNA damage, likely due to apoptosis induction. To exclude direct DNA damage causing an increased in $\gamma \mathrm{H} 2 \mathrm{AX}$, we looked for the induction of ROS in our experimental conditions (single and combined treatments). However, we did not see any ROS production (data not shown), suggesting that apoptosis is likely to be responsible for the observed $\gamma \mathrm{H} 2 \mathrm{AX}$ induction.

The relative lack of toxicity is due to the relatively low dosage of both drugs used, which again underscores the synergistic activity of the combination. In addition, even with the use of higher doses in a clinical setting, the spectrum of the reported side effects of the single drugs (hamatological toxicity for onvansertib and neurotoxicity for paclitaxel) $[43,44]$ are not overlapping, fostering this drug combination. We also reported a synergistic activity when PLK1 inhibitors were combined with both eribulin and PI3K inhibitor. The synergistic effect with eribulin, an anti-mitotic drug with a mechanism of action different from paclitaxel [45], has already been reported in rhabdomyosarcoma [46] and Ewing sarcoma [47]. The latter data reinforce the potentiality of the combination between PLK1 inhibitors and the class of anti-mitotic drugs. The synergistic activity of PLK1 and PI3K inhibition combination has been recently reported in anaplastic thyroid cancer models and was likely to be due to a reducing mitotic slippage and endoreduplication [48]. Finally, considering that PLK1 inhibitor shows activity also in HGSOC, the combination of PLK1 inhibitor and paclitaxel could represent a new option also in this setting, particularly in platinum refracting or relapsing tumors.

\section{Conclusions}

Taken together, our in vitro and in vivo data indicate, for the first time, that PLK1 inhibitors in combination with antimitotic drugs might represent potential therapeutic options for the treatment of mEOC patients. Considering that paclitaxel is already approved for mEOC treatment, this could favor a speedy clinical translation of this combination.

Supplementary Materials: The following are available online at http://www.mdpi.com/2072-6694/12/3/672/s1, Figure S1: cell cycle analysis of mEOC cells. Figure S2: drug combination of onvansertib or volasertib and cisplatin in mEOC cell lines. Figure S3: drug combination of volasertib and paclitaxel or eribulin in mEOC cell lines. Figure S4: drug combination of onvansertib or volasertib and PIK75 in mEOC cell lines. Figure S5: DNA damage and apoptosis kinetics after combination of onvansertib/paclitaxel. Figure S6: effect of the combined treatment onvansertib/eribulin on MCAS cell line. Figure S7: body weight curves in MCAS xenografts. Figure S8: caspase-3 activity assay in EFO27 cell line after combination of onvansertib/paclitaxel. Table S1: Drugs and mechanism of action. Table S2: hits identified with a mean of $\mathrm{T} / \mathrm{C} \leq 0.6$ in the two experiments. Table S3: Mutation status of mEOC cell lines.

Author Contributions: Conceptualization, G.D., L.C. and R.A.; methodology, R.A., R.C., V.R. and M.L.; software, R.A. and R.C.; validation, R.A., L.C., R.C. and V.R.; formal analysis, R.A., L.C., R.C. and V.R.; investigation, R.A., L.C., R.C. and V.R.; resources, R.A., M.L. and L.C.; data curation, R.A., L.C., R.C., V.R. and G.D.; writing-original draft preparation, G.D., R.A., M.L. and L.C.; writing-review and editing, G.D., R.A., L.C., M.L., R.C. and V.R. All authors have read and agreed to the published version of the manuscript.

Funding: This research was funded by the Italian Association for Cancer Research (AIRC), grant number IG 19797 to G.D.

Acknowledgments: The generous contributions of AIRC (The Italian Association for Cancer Research) are grateful acknowledged. We are grateful to Mark Erlander (Trovagene) for providing us onvansertib. A special thanks to Anna and Ennio Galante for supporting the fellowship of RA.

Conflicts of Interest: The authors declare no conflict of interest. 


\section{Abbreviations}

EOC: epithelial ovarian cancer

mEOC: mucinous epithelial ovarian cancer

HGSOC: high grade serous ovarian cancer

RNAi: RNA interference

PLK1: polo-like kinase 1

esiRNA: endoribonuclease-prepared siRNAs

$\gamma \mathrm{H} 2 \mathrm{AX}$ : phospho histone family member $2 \mathrm{AX}$

\section{References}

1. Siegel, R.L.; Miller, K.D.; Jemal, A. Cancer statistics, 2019. CA Cancer J. Clin. 2019, 69, 7-34. [CrossRef] [PubMed]

2. Reid, B.M.; Permuth, J.B.; Sellers, T.A. Epidemiology of ovarian cancer: A review. Cancer Biol. Med. 2017, 14, 9-32. [PubMed]

3. Siegel, R.L.; Miller, K.D.; Jemal, A. Cancer statistics, 2018: Cancer Statistics, 2018. CA Cancer J. Clin. 2018, 68, 7-30. [CrossRef] [PubMed]

4. Lheureux, S.; Braunstein, M.; Oza, A.M. Epithelial ovarian cancer: Evolution of management in the era of precision medicine. CA Cancer J. Clin. 2019, 69, 280-304. [CrossRef]

5. Hess, V.; A’Hern, R.; Nasiri, N.; King, D.M.; Blake, P.R.; Barton, D.P.J.; Shepherd, J.H.; Ind, T.; Bridges, J.; Harrington, K.; et al. Mucinous Epithelial Ovarian Cancer: A Separate Entity Requiring Specific Treatment. J. Clin. Oncol. 2004, 22, 1040-1044. [CrossRef]

6. Shimada, M.; Kigawa, J.; Ohishi, Y.; Yasuda, M.; Suzuki, M.; Hiura, M.; Nishimura, R.; Tabata, T.; Sugiyama, T.; $\mathrm{Kaku}, \mathrm{T}$. Clinicopathological characteristics of mucinous adenocarcinoma of the ovary. Gynecol. Oncol. 2009, 113, 331-334. [CrossRef]

7. Morice, P.; Gouy, S.; Leary, A. Mucinous Ovarian Carcinoma. N. Engl. J. Med. 2019, 380, 1256-1266. [CrossRef]

8. Xu, W.; Rush, J.; Rickett, K.; Coward, J.I.G. Mucinous ovarian cancer: A therapeutic review. Crit. Rev. Oncol. Hematol. 2016, 102, 26-36. [CrossRef]

9. Alexandre, J.; Ray-Coquard, I.; Selle, F.; Floquet, A.; Cottu, P.; Weber, B.; Falandry, C.; Lebrun, D.; Pujade-Lauraine, E. For the GINECO Mucinous advanced epithelial ovarian carcinoma: Clinical presentation and sensitivity to platinum-paclitaxel-based chemotherapy, the GINECO experience. Ann. Oncol. 2010, 21, 2377-2381. [CrossRef]

10. Schiavone, M.B.; Herzog, T.J.; Lewin, S.N.; Deutsch, I.; Sun, X.; Burke, W.M.; Wright, J.D. Natural history and outcome of mucinous carcinoma of the ovary. Am. J. Obstet. Gynecol. 2011, 205, 480.e1-480.e8. [CrossRef]

11. Perren, T.J. Mucinous epithelial ovarian carcinoma. Ann. Oncol. 2016, 27, i53-i57. [CrossRef] [PubMed]

12. Ricci, F.; Affatato, R.; Carrassa, L.; Damia, G. Recent Insights into Mucinous Ovarian Carcinoma. Int. J. Mol. Sci. 2018, 19, 1569. [CrossRef] [PubMed]

13. Australian Ovarian Cancer Study Group; Ryland, G.L.; Hunter, S.M.; Doyle, M.A.; Caramia, F.; Li, J.; Rowley, S.M.; Christie, M.; Allan, P.E.; Stephens, A.N.; et al. Erratum to: Mutational landscape of mucinous ovarian carcinoma and its neoplastic precursors. Genome Med. 2017, 9, 1. [CrossRef] [PubMed]

14. Evans, D.G.R.; Young, K.; Bulman, M.; Shenton, A.; Wallace, A.; Lalloo, F. Probability of BRCA1/2 mutation varies with ovarian histology: Results from screening 442 ovarian cancer families. Clin. Genet. 2008, 73, 338-345. [CrossRef]

15. Chang, K.-L.; Lee, M.-Y.; Chao, W.-R.; Han, C.-P. The status of Her2 amplification and Kras mutations in mucinous ovarian carcinoma. Hum. Genom. 2016, 10, 40. [CrossRef]

16. Gemignani, M.L.; Schlaerth, A.C.; Bogomolniy, F.; Barakat, R.R.; Lin, O.; Soslow, R.; Venkatraman, E.; Boyd, J. Role of KRAS and BRAF gene mutations in mucinous ovarian carcinoma. Gynecol. Oncol. 2003, 90, 378-381. [CrossRef]

17. Henderson, M.C.; Azorsa, D.O. High-Throughput RNAi Screening for the Identification of Novel Targets. In Target Identification and Validation in Drug Discovery: Methods and Protocols; Moll, J., Colombo, R., Eds.; Methods in Molecular Biology; Humana Press: Totowa, NJ, USA, 2013; pp. 89-95. ISBN 978-1-62703-311-4. 
18. Yin, H.; Kassner, M. In Vitro High-Throughput RNAi Screening to Accelerate the Process of Target Identification and Drug Development. In High-Throughput RNAi Screening: Methods and Protocols; Azorsa, D.O., Arora, S., Eds.; Methods in Molecular Biology; Springer New York: New York, NY, USA, 2016; pp. 137-149. ISBN 978-1-4939-6337-9.

19. Carrassa, L.; Chilà, R.; Lupi, M.; Ricci, F.; Celenza, C.; Mazzoletti, M.; Broggini, M.; Damia, G. Combined inhibition of Chk1 and Wee1: In vitro synergistic effect translates to tumor growth inhibition in vivo. Cell Cycle 2012, 11, 2507-2517. [CrossRef]

20. Ricci, F.; Bizzaro, F.; Cesca, M.; Guffanti, F.; Ganzinelli, M.; Decio, A.; Ghilardi, C.; Perego, P.; Fruscio, R.; Buda, A.; et al. Patient-derived ovarian tumor xenografts recapitulate human clinicopathology and genetic alterations. Cancer Res. 2014, 74, 6980-6990. [CrossRef]

21. Restelli, V.; Lupi, M.; Chilà, R.; Vagni, M.; Tarantelli, C.; Spriano, F.; Gaudio, E.; Bertoni, F.; Damia, G.; Carrassa, L. DNA Damage Response Inhibitor Combinations Exert Synergistic Antitumor Activity in Aggressive B-Cell Lymphomas. Mol. Cancer Ther. 2019, 18, 1255-1264. [CrossRef]

22. Nicoletti, M.I.; Valoti, G.; Giannakakou, P.; Zhan, Z.; Kim, J.-H.; Lucchini, V.; Landoni, F.; Mayo, J.G.; Giavazzi, R.; Fojo, T. Expression of $\beta$-Tubulin Isotypes in Human Ovarian Carcinoma Xenografts and in a Sub-Panel of Human Cancer Cell Lines from the NCI-Anticancer Drug Screen: Correlation with Sensitivity to Microtubule Active Agents. Clin. Cancer Res. 2001, 7, 2912-2922.

23. Pisano, C.; Greggi, S.; Tambaro, R.; Losito, S.; Iodice, F.; Maio, M.D.; Ferrari, E.; Falanga, M.; Formato, R.; Iaffaioli, V.R.; et al. Activity of Chemotherapy in Mucinous Epithelial Ovarian Cancer: A Retrospective Study. Anticancer Res. 2005, 5, 3501-3505.

24. Barr, F.A.; Silljé, H.H.W.; Nigg, E.A. Polo-like kinases and the orchestration of cell division. Nature Rev. Mol. Cell Biol. 2004, 5, 429-441. [CrossRef] [PubMed]

25. Eckerdt, F.; Yuan, J.; Strebhardt, K. Polo-like kinases and oncogenesis. Oncogene 2005, 24, 267-276. [CrossRef] [PubMed]

26. Takai, N.; Miyazaki, T.; Fujisawa, K.; Nasu, K.; Hamanaka, R.; Miyakawa, I. Expression of polo-like kinase in ovarian cancer is associated with histological grade and clinical stage. Cancer Lett. 2001, 164, 41-49. [CrossRef]

27. Weichert, W.; Denkert, C.; Schmidt, M.; Gekeler, V.; Wolf, G.; Köbel, M.; Dietel, M.; Hauptmann, S. Polo-like kinase isoform expression is a prognostic factor in ovarian carcinoma. Br. J. Cancer 2004, 90, 815-821. [CrossRef]

28. Liu, X. Targeting Polo-Like Kinases: A Promising Therapeutic Approach for Cancer Treatment. Transl. Oncol. 2015, 8, 185-195. [CrossRef]

29. Liu, Z.; Sun, Q.; Wang, X. PLK1, A Potential Target for Cancer Therapy. Transl. Oncol. 2017, 10, $22-32$. [CrossRef]

30. Weiß, L.; Efferth, T. Polo-like kinase 1 as target for cancer therapy. Exp. Hematol. Oncol. 2012, 1, 38. [CrossRef]

31. Gutteridge, R.E.A.; Ndiaye, M.A.; Liu, X.; Ahmad, N. Plk1 Inhibitors in Cancer Therapy: From Laboratory to Clinics. Mol. Cancer Ther. 2016, 15, 1427-1435. [CrossRef]

32. Hao, Z.; Kota, V. Volasertib for AML: Clinical use and patient consideration. OncoTargets Ther. 2015, 8, 1761. [CrossRef]

33. Pujade-Lauraine, E.; Selle, F.; Weber, B.; Ray-Coquard, I.-L.; Vergote, I.; Sufliarsky, J.; Del Campo, J.M.; Lortholary, A.; Lesoin, A.; Follana, P.; et al. Volasertib Versus Chemotherapy in Platinum-Resistant or -Refractory Ovarian Cancer: A Randomized Phase II Groupe des Investigateurs Nationaux pour l'Etude des Cancers de l'Ovaire Study. J. Clin. Oncol. 2016, 34, 706-713. [CrossRef] [PubMed]

34. Bu, Y.; Yang, Z.; Li, Q.; Song, F. Silencing of Polo-Like Kinase (Plk) 1 via siRNA Causes Inhibition of Growth and Induction of Apoptosis in Human Esophageal Cancer Cells. OCL 2008, 74, 198-206. [CrossRef] [PubMed]

35. Reagan-Shaw, S.; Ahmad, N. Silencing of polo-like kinase (Plk) 1 via siRNA causes induction of apoptosis and impairment of mitosis machinery in human prostate cancer cells: Implications for the treatment of prostate cancer. FASEB J. 2005, 19, 611-613. [CrossRef] [PubMed]

36. Spankuch-Schmitt, B.; Bereiter-Hahn, J.; Kaufmann, M.; Strebhardt, K. Effect of RNA Silencing of Polo-Like Kinase-1 (PLK1) on Apoptosis and Spindle Formation in Human Cancer Cells. JNCI J. Natl. Cancer Inst. 2002, 94, 1863-1877. [CrossRef]

37. Gjertsen, B.T.; Schöffski, P. Discovery and development of the Polo-like kinase inhibitor volasertib in cancer therapy. Leukemia 2015, 29, 11-19. [CrossRef] [PubMed] 
38. Rudolph, D.; Impagnatiello, M.A.; Blaukopf, C.; Sommer, C.; Gerlich, D.W.; Roth, M.; Tontsch-Grunt, U.; Wernitznig, A.; Savarese, F.; Hofmann, M.H.; et al. Efficacy and Mechanism of Action of Volasertib, a Potent and Selective Inhibitor of Polo-Like Kinases, in Preclinical Models of Acute Myeloid Leukemia. J. Pharmacol. Exp. Ther. 2015, 352, 579-589. [CrossRef]

39. Valsasina, B.; Beria, I.; Alli, C.; Alzani, R.; Avanzi, N.; Ballinari, D.; Cappella, P.; Caruso, M.; Casolaro, A.; Ciavolella, A.; et al. NMS-P937, an Orally Available, Specific Small-Molecule Polo-like Kinase 1 Inhibitor with Antitumor Activity in Solid and Hematologic Malignancies. Mol. Cancer Ther. 2012, 11, 1006-1016. [CrossRef]

40. Raab, M.; Krämer, A.; Hehlgans, S.; Sanhaji, M.; Kurunci-Csacsko, E.; Dötsch, C.; Bug, G.; Ottmann, O.; Becker, S.; Pachl, F.; et al. Mitotic arrest and slippage induced by pharmacological inhibition of Polo-like kinase 1. Mol. Oncol. 2015, 9, 140-154. [CrossRef]

41. Chou, Y.-S.; Yen, C.-C.; Chen, W.-M.; Lin, Y.-C.; Wen, Y.-S.; Ke, W.-T.; Wang, J.-Y.; Liu, C.-Y.; Yang, M.-H.; Chen, T.-H.; et al. Cytotoxic mechanism of PLK1 inhibitor GSK461364 against osteosarcoma: Mitotic arrest, apoptosis, cellular senescence, and synergistic effect with paclitaxel. Int. J. Oncol. 2016, 48, 1187-1194. [CrossRef]

42. Noack, S.; Raab, M.; Matthess, Y.; Sanhaji, M.; Krämer, A.; Győrffy, B.; Kaderali, L.; El-Balat, A.; Becker, S.; Strebhardt, K. Synthetic lethality in CCNE1-amplified high grade serous ovarian cancer through combined inhibition of Polo-like kinase 1 and microtubule dynamics. Oncotarget 2018, 9, 25842. [CrossRef]

43. Weiss, G.J.; Jameson, G.; Von Hoff, D.D.; Valsasina, B.; Davite, C.; Di Giulio, C.; Fiorentini, F.; Alzani, R.; Carpinelli, P.; Di Sanzo, A.; et al. Phase I dose escalation study of NMS-1286937, an orally available Polo-Like Kinase 1 inhibitor, in patients with advanced or metastatic solid tumors. Investig. New Drugs 2018, 36, 85-95. [CrossRef] [PubMed]

44. Rowinsky, E.K.; Chaudhry, V.; Cornblath, D.R.; Donehower, R.C. Neurotoxicity of Taxol. J. Natl. Cancer Inst. Monogr. 1993, 107-115.

45. Dybdal-Hargreaves, N.F.; Risinger, A.L.; Mooberry, S.L. Eribulin mesylate: Mechanism of action of a unique microtubule-targeting agent. Clin. Cancer Res. 2015, 21, 2445-2452. [CrossRef] [PubMed]

46. Hugle, M.; Belz, K.; Fulda, S. Identification of synthetic lethality of PLK1 inhibition and microtubule-destabilizing drugs. Cell Death Differ. 2015, 22, 1946-1956. [CrossRef] [PubMed]

47. Weiß, L.M.; Hugle, M.; Fulda, S. Eribulin alone or in combination with the PLK1 inhibitor BI 6727 triggers intrinsic apoptosis in Ewing sarcoma cell lines. Oncotarget 2017, 8, 52445-52456. [CrossRef] [PubMed]

48. De Martino, D.; Yilmaz, E.; Orlacchio, A.; Ranieri, M.; Zhao, K.; Di Cristofano, A. PI3K blockage synergizes with PLK1 inhibition preventing endoreduplication and enhancing apoptosis in anaplastic thyroid cancer. Cancer Lett. 2018, 439, 56-65. [CrossRef]

(C) 2020 by the authors. Licensee MDPI, Basel, Switzerland. This article is an open access article distributed under the terms and conditions of the Creative Commons Attribution (CC BY) license (http://creativecommons.org/licenses/by/4.0/). 